\title{
Down-regulation of B-Cell Translocation Gene 1 by Promoter Methylation in Colorectal Carcinoma
}

\author{
YOON YANG JUNG ${ }^{1}$, JI-YOUN SUNG ${ }^{2}$, JI-YE KIM ${ }^{3,4}$ and HYUN-SOO KIM ${ }^{3}$ \\ ${ }^{1}$ Department of Pathology, Myongji Hospital, Goyang, Republic of Korea; \\ ${ }^{2}$ Department of Pathology, Kyung Hee University School of Medicine, Seoul, Republic of Korea; \\ ${ }^{3}$ Department of Pathology, Severance Hospital, Yonsei University College of Medicine, Seoul, Republic of Korea; \\ ${ }^{4}$ Department of Pathology and Translational Genomics, Samsung Medical Center, \\ Sungkyunkwan University School of Medicine, Seoul, Republic of Korea
}

\begin{abstract}
Background: B-cell translocation gene 1 (BTG1) acts as a tumour suppressor in human malignancies. However, the precise mechanism of BTG1 down-regulation in colorectal carcinoma (CRC) remains unclear. We analyzed BTGI expression in CRC cell lines and tissues and investigated the mechanism underlying the observed alterations. Materials and Methods: Real-time polymerase chain reaction (PCR) and western blot analyses were performed to analyze BTGI expression in CRC cell lines. The methylation status of the BTG1 promoter region in cell lines was determined by methylation-specific PCR, and the effect of demethylation on BTG1 expression was explored with 5-aza-deoxycytidine treatment. BTG1 protein expression in CRC tissue samples was evaluated using immunostaining. Results: $C R C$ cell lines and tissue samples expressed lower levels of BTGI compared to controls, and BTG1 levels were significantly lower in metastatic than primary CRC. In BTG1-down-regulated CRC cell lines, the BTG1 promoter was highly methylated, and 5aza-deoxycytidine significantly restored BTG1 expression. Conclusion: BTG1 down-regulation in CRC occurs through epigenetic repression, which is involved in the development and progression of $C R C$.
\end{abstract}

Colorectal carcinoma (CRC) is one of the most frequently diagnosed malignancies in the developed world. It is the third most common cancer and the fourth leading cause of cancer-related death in the Republic of Korea $(1,2)$. In Western countries, CRC is the fourth most common cancer

Correspondence to: Hyun-Soo Kim, Department of Pathology, Severance Hospital, Yonsei University College of Medicine, 50-1 Yonsei-ro, Seodaemun-gu, Seoul 03722, Republic of Korea. Tel: +82 222281794, +82 23620860, e-mail: hyunsookim@yuhs.ac

Key Words: Colon, colorectal carcinoma, B-cell translocation gene 1, down-regulation, promoter methylation. and the second leading cause of cancer-related death (3-5). Despite advances in operative techniques and improvements in chemotherapeutic agents, the prognosis of patients with CRC remains poor because of local recurrence, lymph node metastasis, and distant metastasis. It is important for pathologists to provide accurate pathological and molecular features of tumours, that may affect the treatment decisions of oncologists and surgeons.

Cellular differentiation, apoptosis, and proliferation are cell cycle-dependent processes (6). Many proto-oncogenes and tumour-suppressor genes are directly involved in or function as major factors in cell-cycle regulation. Aberrant expression of these genes results in deregulation of the cell cycle, abnormal cellular proliferation, and tumour development (7). Impaired cell-cycle regulation is closely linked to tumour development and progression $(8,9)$. B-Cell translocation gene 1 (BTGI) was originally identified as a translocation partner of the $c-m y c$ gene in B-cell chronic lymphocytic leukaemia. BTG1 is a member of the mammalian BTG/Tob family of proteins that are involved in regulation of cell-cycle progression, inhibition of cellular proliferation, promotion of apoptosis, and stimulation of cellular differentiation in various cell types (10-13). Exogenous expression of BTG1 was found to reduce proliferation through $G_{1}$ arrest and apoptosis in murine fibroblasts (14). Similarly, the BTG1 expression levels is inversely correlated with the proliferative capacity of murine microglial cells (15). Moreover, enhanced BTG1 expression promotes the differentiation of neural stem cells and germ cells $(16,17)$.

Based on these characteristics, BTGl is currently considered to be a tumour-suppressor gene. Experiments in induction of gene expression showed that BTGI mediated apoptosis and negatively regulated cellular proliferation, cellto-cell adhesion, migration, and invasion in several human malignancies (18-24). However, the role of BTGl in the development and progression of CRC is yet to be elucidated. 
Moreover, the precise molecular mechanisms of action of $B T G 1$ in CRC remain unclear.

In this study, BTGl expression was evaluated and compared between CRC and normal colonic epithelial cell lines in order to clarify the role of $B T G 1$ in colorectal carcinogenesis. We further analysed the promoter methylation status of the BTGl promoter region in CRC cell lines to understand the mechanism responsible for any observed alterations in BTG1 expression, and compared the BTG1 expression status before and after demethylation treatment. In addition, we compared BTG1 expression between primary and metastatic CRC tissue samples. Our results indicate that promoter methylation is one of the mechanisms contributing to the down-regulation of BTGI in CRC, suggesting its involvement in the development and progression of CRC.

\section{Materials and Methods}

Cell culture and treatment. The human normal colonic epithelial cell line CCD 841 CoTr and the human CRC cell lines HCT 116, HT-29, SW 480, and SW 620 were purchased from the American Type Culture Collection (Manassas, VA, USA) and maintained in Dulbecco's modified Eagle's medium (DMEM) or Roswell Park Memorial Institute (RPMI) 1640 medium supplemented with $10 \%$ heat-inactivated foetal bovine serum, penicillin $(100 \mathrm{U} / \mathrm{ml})$, and streptomycin $(100 \mu \mathrm{g} / \mathrm{ml}$; Gibco, Life Technologies, Grand Island, NY, USA) (25). All cell lines were cultured at $37^{\circ} \mathrm{C}$ in a humidified atmosphere of $5 \%$ carbon dioxide. The demethylating agent 5-aza-deoxycytidine (5-aza-CdR) was obtained from Sigma-Aldrich (St. Louis, MO, USA), dissolved in phosphate-buffered saline (PBS) to a concentration of $50 \mathrm{mg} / \mathrm{ml}$ as a stock solution, and stored at $-20^{\circ} \mathrm{C}$ until use.

Cell lines were seeded in 6-well plates at a density of $5 \times 10^{5}$ cells $/ \mathrm{mL}$ in the media and treated with $1 \mu \mathrm{M}$ (final concentration) of 5-aza-CdR for $72 \mathrm{~h}$. Freshly prepared 5-aza-CdR was replaced every $24 \mathrm{~h}$ and cells were harvested at $96 \mathrm{~h}$ after initial treatment. Control cultures were treated under similar experimental conditions in the absence of 5-aza-CdR (final concentration (PBS only).

cDNA synthesis. RNase-free DNase I treatment was carried out to remove contaminating genomic DNA from purified total RNA obtained from the cell lines. Isolated total RNA was diluted to $1 \mathrm{mg} / \mathrm{ml}$ with sterile diethylpyrocarbonate-treated water and $2.5 \mathrm{ml}$ was added to reactions containing $1 \times$ DNase I buffer and $1 \mathrm{U}$ DNase I (final volume, $10 \mathrm{ml}$ ). After incubation at $37^{\circ} \mathrm{C}$ for $30 \mathrm{~min}$, the reactions were stopped at $70^{\circ} \mathrm{C}$ for $10 \mathrm{~min}$. DNase I-treated RNA was reversetranscribed into first-strand cDNA using random primers. DNase Itreated RNA $(1 \mu \mathrm{g})$ and random primers $(250 \mathrm{ng})$ were mixed in a 0.5 $\mathrm{ml}$ polymerase chain reaction (PCR) tube and brought to $11 \mathrm{ml}$ with sterile diethylpyrocarbonate-treated water, heated at $65^{\circ} \mathrm{C}$ for $5 \mathrm{~min}$, and chilled quickly on ice. Other reagents were added to the $20-\mathrm{ml}$ reaction volume at the following final concentrations: $1 \times$ First-Strand Buffer, $10 \mathrm{mM}$ dithiothreitol, $0.5 \mathrm{mM}$ each dNTP, and $200 \mathrm{U}$ Superscript II Reverse Transcriptase (Invitrogen, Carlsbad, CA, USA). Reactions were incubated at $42^{\circ} \mathrm{C}$ for $1 \mathrm{~h}$, heated to $70^{\circ} \mathrm{C}$ for $10 \mathrm{~min}$, and the products were stored at $-20^{\circ} \mathrm{C}$.

Quantitative reverse-transcriptase $(q R T)-P C R$. Total RNA was isolated using TRI Reagent (Molecular Research Center, Cincinnati,
$\mathrm{OH}, \mathrm{USA}$ ) and used for cDNA synthesis with a ReverTra Ace qPCR RT kit (Toyobo, Osaka, Japan). The amount of cDNA was determined spectrophotometrically. The cDNA was used for qRTPCR using the Bio-Rad CFX96 Real-Time PCR Detection System (Bio-Rad Laboratories, Hercules, CA, USA) with a C1000 Thermal Cycler (Bio-Rad Laboratories). PCR was carried out in a $20-\mu 1$ reaction containing $0.5 \mu \mathrm{M}$ of each primer, $10 \mu \mathrm{l}$ of $2 \times$ SsoAdvanced SYBR Green Supermix (Bio-Rad Laboratories), and $2 \mu$ of template DNA. PCRs for BTG1 and $\beta$-actin were initiated with a denaturing step at $95^{\circ} \mathrm{C}$ for $3 \mathrm{~min}$, followed by 40 cycles at $95^{\circ} \mathrm{C}$ for $30 \mathrm{~s}$ and $60^{\circ} \mathrm{C}$ for $30 \mathrm{~s}$. Amplification patterns were analysed and threshold cycle numbers $(\mathrm{Ct})$ for each sample were determined using CFX Manager Software (Bio-Rad Laboratories). The $\Delta \Delta \mathrm{Ct}$ method was used to calculate relative target gene expression after normalization to expression of $\beta$-actin (24). The primer sequences used for BTG1 were as follows: forward: 5'-CAA GGG ATC GGG TTA CCG TTG T-3'; reverse: 5'-AGC CAT CCT CTC CAA TTC TGT AGG-3'. The $\Delta \Delta \mathrm{Ct}$ method was used to calculate relative target gene expression after normalization to expression of $\beta$-actin (26). Amplification of the target gene was confirmed by melting-curve analysis and target amplicon size was confirmed by agarose gel electrophoresis. Each sample was assayed in triplicate.

Western blot analysis. Whole-cell lysates were prepared in radioimmunoprecipitation assay buffer $(50 \mathrm{mM}$ Tris-hydrogen chloride, $\mathrm{pH} 8,150 \mathrm{mM}$ sodium chloride, $1 \% \mathrm{NP}-40,0.5 \%$ sodium deoxycholate, and $0.1 \%$ sodium dodecyl sulphate) containing protease inhibitors (cOmplete Protease Inhibitor Cocktail Tablet; Roche Applied Science, Basel, Switzerland), and cleared by microcentrifugation $\left(10,000 \times \mathrm{g}\right.$ for $20 \mathrm{~min}$ at $\left.4^{\circ} \mathrm{C}\right)$. The resulting lysate was assessed for protein concentration, and 20-30 $\mu \mathrm{g}$ of each protein sample was resolved using $12 \%$ sodium dodecyl sulphateprotein gel electrophoresis gel (Bio-Rad Laboratories) and electroblotted onto nitrocellulose membranes (GE Healthcare, Little Chalfont, UK). After 1-h incubation in blocking solution (5\% nonfat dry milk in Tris-buffered saline with Tween; Pierce, Rockford, IL, USA), the membranes were exposed to the following appropriate primary antibodies overnight at $4^{\circ} \mathrm{C}$ : anti-BTG1 (polyclonal, diluted 1:200; Abcam, Cambridge, MA, USA) and anti$\alpha$-tubulin (diluted 1:500; Abcam). The blots were washed three times in Tris-buffered saline with Tween and incubated with the horseradish peroxidase-conjugated secondary antibody (Cell Signaling Technology, Beverly, MA, USA) for $1 \mathrm{~h}$ at room temperature. Protein bands were visualized using enhanced chemiluminescence reagent (iNtRON Biotechnology, Seongnam, Republic of Korea).

Methylation-specific PCR. Genomic DNA was extracted from the cell lysate using a NucleoSpin Tissue kit (Macherey-Nagel, Dueren, Germany) and treated with sodium bisulphite using an EZ DNA Methylation Kit (Zymo Research, Irvine, CA, USA). During the modification, unmethylated cytosines of the genomic DNA are converted to uracils, while methylated cytosines remain unchanged. The bisulphite-modified DNA was subjected to PCR using primer pairs that specifically amplified either methylated or unmethylated sequences of BTG1. The following primers specific for methylated BTG1 were used (27): MSP1 (-149 to -289), 5'-GTT TTT AAG TTA AAA GGA AGG AAG TC-3' (sense) and 5'-ATA TCA AAA AAT ATT AAA AAT CAC GCA-3' (antisense); MSP2 (-517 to -645), 5'TTT GAG GAG TTA GTT ATC GAG ATT C-3' (sense) and 5'-AAA 
TAA ATA AAA ACC GCC TAA CG-3' (antisense). The following primers specific for unmethylated $B T G 1$ were used: USP1 (-149 to -289), 5'-GTT TTT AAG TTA AAA GGA AGG AAG TTG T-3' (sense) and 5'-ATA TCA AAA ATA TTA AAA ATC ACA CA-3' (antisense); USP2 (-517 to -645), 5'-TGA GGA GTT AGT TAT TGA GAT TTG G-3' (sense) and 5'-AAA TAA ATA AAA ACC ACC TAA CAC A-3' (antisense). Methylation-specific PCR was performed in $20-\mu 1$ mixtures for 40 cycles using HotStarTaq DNA polymerase (Qiagen, Hilden, Germany). The same unconverted genomic DNAs were used in the PCR assays as negative controls.

Tissue specimens. This study was reviewed and approved by the Institutional Review Board of Myongji Hospital, Goyang, Republic of Korea (2017-11-001). CRC tissue samples were obtained from 114 consecutive patients who underwent surgery for primary adenocarcinoma of the colorectum. Twenty normal colonic mucosa samples obtained from patients without CRC were used as controls. Corresponding tissue samples from hepatic CRC metastases were also obtained from 79 patients who met the criteria for hepatic resection with curative intent $(25,28-30)$. In this study. no patient underwent preoperative neoadjuvant chemotherapy or neoadjuvant concurrent chemoradiation therapy.

The tissues resected by surgeons were initially examined by pathologists before fixation in $10 \%$ neutral-buffered formalin. After fixation for $12-24 \mathrm{~h}$, the tissues were thoroughly examined macroscopically and sectioned. After processing with an automatic tissue processor, the sections were embedded in paraffin blocks Four-micrometre-thick slices were cut from each formalin-fixed, paraffin-embedded (FFPE) tissue block using a rotary microtome and stained with hematoxylin and eosin using an automatic staining instrument. After staining, the slides were covered with a glass coverslip and sent to two Board-certified pathologists. The pathologists independently examined all available hematoxylin and eosin-stained slides by light microscopy, made definitive pathological diagnoses, and selected the most representative slide from each case for immunohistochemical staining.

Immunohistochemical staining. BTG1 protein expression was assessed by immunohistochemistry using the Bond Polymer Intense Detection System (Vision BioSystems, Mount Waverley, Victoria, Australia) following the manufacturer's instructions $(25,28,31$ 38). In brief, the 4- $\mu \mathrm{m}$ sections of FFPE tissue were deparaffinized with Bond Dewax Solution (Vision BioSystems) and an antigen retrieval procedure was performed using Bond Epitope Retrieval Solution (Vision BioSystems) for $30 \mathrm{~min}$ at $100^{\circ} \mathrm{C}$. Endogenous peroxidases were quenched with hydrogen peroxide for $5 \mathrm{~min}$. Sections were incubated for $15 \mathrm{~min}$ at ambient temperature with rabbit polyclonal antibody to BTG1 (diluted 1:100; Abcam). A biotin-free polymeric horseradish peroxidase-linker antibody conjugate system was used with a Bond-maX automatic slide stainer (Vision BioSystems), and visualization was performed using $1 \mathrm{mM}$ 3,3'-diaminobenzidine, $50 \mathrm{mM}$ Tris-hydrogen chloride buffer ( $\mathrm{pH} 7.6$ ), and $0.006 \%$ hydrogen peroxide. Sections were counterstained with hematoxylin. To minimize inter-assay variation, positive and negative control samples were included in each run. The positive control was normal colonic tissue. The negative control was prepared by substituting non-immune serum for the primary antibody; no detectable staining was evident. Interpretation of immunohistochemical staining. Immunostaining was analysed by two independent pathologists. The BTG1 staining intensity was graded as: 0 : absent, 1: weak, 2 : intermediate, or 3 : strong. The staining proportion was graded as: $0: 0 \%, 1: 1-49 \%, 2$ : $50-74 \%$, or $3: 75-100 \%$. The subcellular location of BTG1-positive signals (nuclear or cytoplasmic) was also estimated. The final score was calculated as the product of the staining intensity and proportion scores, resulting in final scores of 0 (negative), 1-2 (weak), and 3-9 (positive) $(25,27,34,35,39,40)$. Disagreements between the two pathologists were resolved by consensus.

Statistical analysis. We used the Wilcoxon test to compare the expression levels of BTG1 mRNA before and after 5-aza-CdR treatment. Chi-squared or Fisher's exact tests were performed to compare BTG1 immunoreactivity among the normal colonic mucosa, primary CRC, and metastatic CRC tissue samples. Statistical analyses were performed using PASW Statistics for Windows (version 18.0; Armonk, NY, USA). $p$-Values less than 0.05 were considered statistically significant.

\section{Results}

Mechanism contributing to BTG1 down-regulation. BTG1 protein was expressed at different levels in different CRC cell lines. The CRC cell lines HCT 116, HT-29, SW 480, and SW 620 exhibited significantly reduced BTG1 protein expression compared to the normal colonic epithelial cell line CCD 841 CoTr. Consistent with these findings, HCT 116, HT-29, and SW 480 cells expressed significantly lower levels of BTG1 mRNA than CCD 841 CoTr cells. We recently observed that $B T G 1$ promoter methylation was increased in ovarian carcinoma cell lines and that BTG1 expression was restored after treatment with the demethylating agent 5-aza-CdR (40). Based on this previous observation, we assumed that promoter methylation might explain the reduced expression of BTGl in CRC cell lines. We observed increased methylation of the BTG1 promoter region (MSP1) in HCT 116 and SW 480 cells (Figure 1A), indicating a possible mechanism for the reduced BTG1 expression in these cell lines.

To further investigate the effect of promoter methylation on BTG1 down-regulation, we treated these two cell lines with the demethylating agent 5-aza-CdR. Treatment with 5-azaCdR significantly reduced $B T G 1$ promoter methylation in HCT 116 and SW 480 cells (Figure 1B). This reduction was associated with significant restoration of BTGl mRNA expression, with a 4.04-fold increase in HCT 116 cells $(\mathrm{p}<0.001)$ and a 3.72-fold increase in SW 480 cells $(\mathrm{p}<0.001)$ compared to the respective pre-treatment levels (Figure 1C). The restorative effect of 5-aza-CdR on BTG1 expression in HCT 116 cells was confirmed at the protein level (Figure 1D). These findings indicate that promoter methylation is responsible for reduced BTG1 expression in CRC cells.

Reduced BTG1 expression in CRC tissue samples. Representative photomicrographs of BTG1 immunostaining are shown in Figure 1E. BTG1 immunoreactivity was observed in 
A

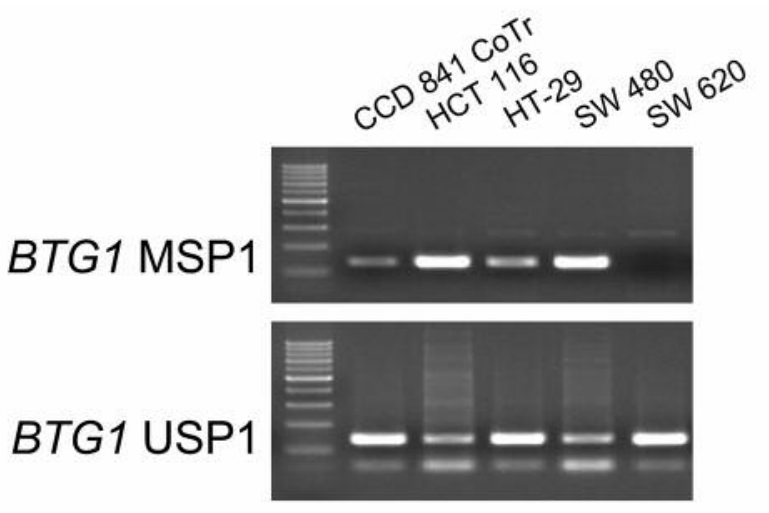

BTG1 MSP2

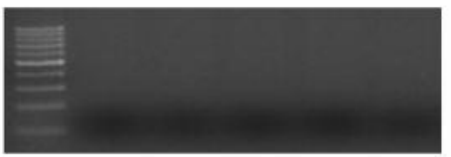

BTG1 USP2

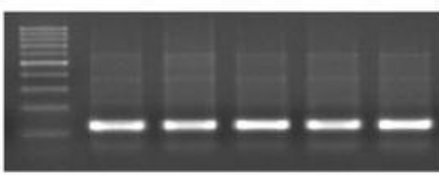

C

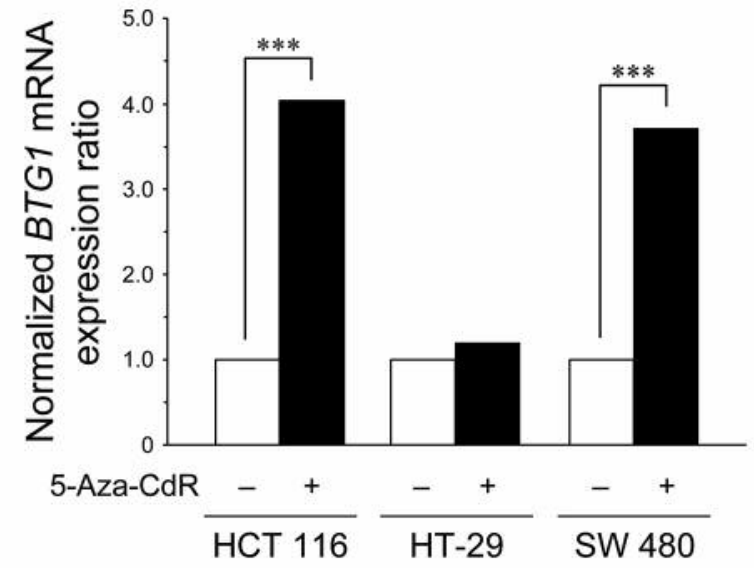

B
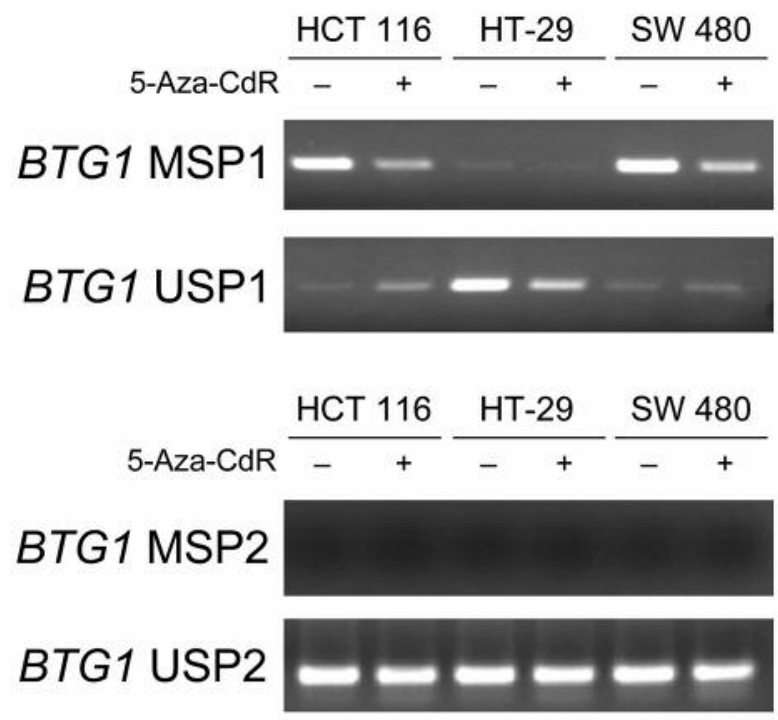

D

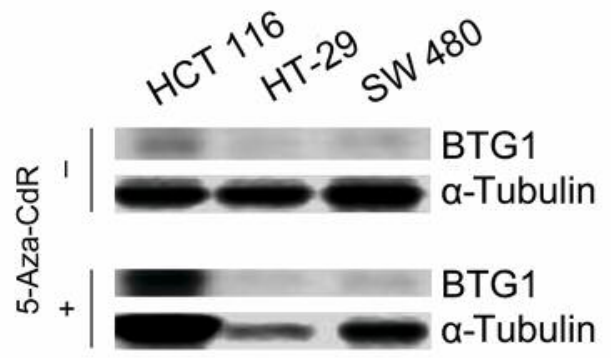

E

Normal colonic mucosa

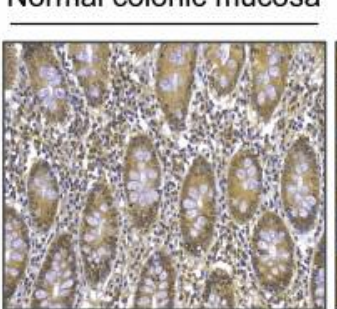

Preserved BTG1 expression
Primary colorectal carcinoma

Metastatic colorectal carcinoma

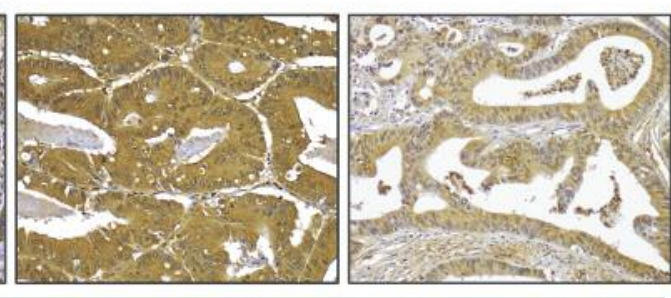

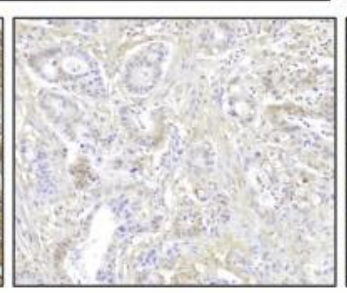

Reduced BTG1 expression

Figure 1. Mechanism contributing to reduced B-cell translocation gene 1 (BTG1) expression in colorectal carcinoma (CRC) cell lines. A: Methylation status of the BTG1 gene promoter region as analysed by methylation-specific polymerase chain reaction. MSP: Methylation-specific polymerase chain reaction primer; USP: unmethylation-specific polymerase chain reaction primer. B: Effect of treatment with the demethylating agent 5-azadeoxycytidine (5-aza-CdR) on BTG1 promoter methylation. C: Restoration of BTG1 mRNA expression by demethylation using 5-aza-CdR*** $p<0.001$. D: Western blot for BTG1 before and after treatment with the demethylating agent. E: BTG1 protein expression in normal colonic mucosa and CRC tissue samples. Original magnification: $E, \times 100$. 
Jung et al: BTG1 Down-regulation in CRC

Table I. B-Cell translocation gene 1 (BTG1) immunoreactivity in colorectal carcinoma (CRC) and normal colonic tissue samples.

\begin{tabular}{lccccc}
\hline Category & Total, $\mathrm{n}$ & \multicolumn{3}{c}{ BTG1 immunoreactivity, $\mathrm{n}(\%)$} & \multirow{2}{*}{$p$-Value } \\
\cline { 3 - 5 } & & Negative & Weak & Positive \\
\hline Normal mucosa $^{\mathrm{a}}$ & 20 & $0(0.0)$ & $8(40.0)$ & $12(60.0)$ & \\
Primary CRC & 114 & $30(26.3)$ & $32(28.1)$ & $52(45.6)$ & $0.036^{\mathrm{a}}$ \\
Metastatic CRC & 79 & $35(44.3)$ & $22(27.8)$ & $22(27.8)$ & $<0.001^{\mathrm{b}}, 0.004^{\mathrm{c}}$ \\
\hline
\end{tabular}

${ }^{\mathrm{a}}$ Versus normal mucosa; ${ }^{\mathrm{b}}$ versus normal mucosa; ${ }^{\mathrm{c}}$ versus primary CRC.

the cytoplasm, although a few tumour cells with strong cytoplasmic BTG1 expression exhibited faint nuclear BTG1 immunoreactivity. Patchy and weak BTG1 immunoreactivity was identified in the extracellular matrix. Diffuse and moderateto-strong cytoplasmic BTG1 immunoreactivity was observed in 12 out of the $20(60.0 \%)$ normal colonic tissue samples. In contrast, 32 (28.1\%) out of the 114 primary CRC tissue samples exhibited weak BTG1 expression, and no BTG1 expression was detected in $30(26.3 \%)$ primary CRC tissue samples. Overall, BTG1 expression in primary CRC tissue samples was significantly decreased compared to that in normal colonic tissue samples ( $p=0.036$; Table I). Moreover, BTG1 expression was significantly reduced in metastatic CRC tissue samples compared to that in primary CRC tissue samples ( $p=0.004$; Table I). Fifty-seven (72.2\%) out of the 79 metastatic CRC tissue samples exhibited weak (22/79) or no (35/79) BTG1 expression.

\section{Discussion}

In this study, we analyzed the mRNA and protein expression of BTG1 in CRC cell lines and tissue samples. We found that CRC cell lines displayed reduced BTG1 expression compared to the normal colonic epithelial cell line. Consistent with these findings, more than half $(54.4 \%)$ of the primary CRC tissue samples showed reduced BTG1 immunoreactivity. The difference in BTG1 expression between primary CRC and normal colonic tissues was significant, indicating that BTG1 is a potential diagnostic biomarker for CRC. In addition, reduced BTG1 expression was observed in $72.2 \%$ of metastatic CRC tissue samples, with a significant difference in BTG1 expression between metastatic and primary CRC tissue samples. Overall, our results indicate that BTGl downregulation is associated with the development and progression of CRC, highlighting BTG1 as a novel therapeutic target for the treatment of patients with CRC.

To investigate the regulatory mechanisms of BTG1 expression, we focused on the promoter methylation status in CRC cells. In CRC cells with BTGl down-regulation, the $B T G 1$ promoter was highly methylated, and demethylation restored BTG1 mRNA and protein expression. These findings suggest that epigenetic mechanisms are responsible for the repression of $B T G 1$ expression in CRC cells, and that the down-regulation of BTGl expression in CRC cell lines is a result of aberrant promoter hypermethylation. In line with these findings, we recently demonstrated that BTG1 mRNA and protein expression levels are reduced in ovarian carcinoma cell lines (40), and that BTG1 promoter methylation was reduced and the BTG1 expression level was restored after treatment with 5-aza-CdR. Reduced BTG1 expression was also observed in gastric carcinoma, in which BTG1 was found to be silenced via promoter hypermethylation (27). Taken together, these results provide new insight concerning the alterations of BTG1 expression. We suggest that the therapeutic targeting of BTG1 is a potential strategy for the treatment of CRC.

However, some experimental data on BTG1 promoter methylation are in conflict with the above findings. Gastric carcinoma and hepatocellular carcinoma cell lines did not exhibit BTG1 promoter hypermethylation (41). These conflicting findings regarding promoter methylation status may be related to the differences in organs and cell types, differences in methods used to analyse methylation status, and the inadequacy of a single model for explaining the complex process of carcinogenesis. Furthermore, down-regulation of BTG1 expression has also been linked to alternative molecular regulatory mechanisms such as microRNAs in renal cell carcinoma and prostate carcinoma $(42,43)$. Taken together, these findings indicate that promoter hypermethylation may be partially responsible for repressed BTG1 expression.

Data on BTG1 expression in other malignancies support the notion that BTG1 may function as a tumour suppressor. BTG1 protein expression in renal cell carcinoma tissues was found to be significantly lower than that in normal kidney tissue (22). BTG1-overexpressing renal cell carcinoma cells, obtained by stable transfection of BTG1 cDNA, had a significantly higher apoptotic rate and lower invasiveness than control cells, which were accompanied by reduced Bcell lymphoma 2 and matrix metalloproteinase expression levels. These findings indicate that BTG1 might inhibit cellular proliferation by reducing B-cell lymphoma 2 
expression and can modulate the invasion and metastasis of cancer cells by down-regulating matrix metalloproteinase. Similarly, BTG1-transfected breast carcinoma cell xenografts had a significantly smaller tumour size, lower cell density, and higher degree of tumour necrosis than untransfected xenografts in control mice (24), indicating that BTG1 overexpression mediates the inhibition of xenograft formation and breast carcinoma cell growth in vivo. In addition, BTG1-transfected hepatocellular carcinoma cells (20), non-small cell lung carcinoma cells (21), and nasopharyngeal carcinoma cells (19) exhibited more frequent $\mathrm{G}_{0} / \mathrm{G}_{1}$ arrest, higher apoptotic rates, and significantly lower invasive capabilities than corresponding untransfected cells.

In terms of clinical significance, BTG1 down-regulation has been linked with aggressive biological behaviour. In gastric carcinoma, reduced BTG1 expression was associated with deeper tumour invasion, lymphovascular invasion, lymph node metastasis, and advanced stage (27). BTG1 mRNA expression in International Federation of Gynecology and Obstetrics (FIGO) stage I-II ovarian carcinomas was reported to be higher than that in FIGO stage III-IV ovarian carcinomas (44). Furthermore, reduced BTG1 expression was significantly associated with adverse clinicopathological parameters and reduced survival in carcinomas of the breast (45), oesophagus (18), liver (46), lung (21), nasopharynx (19), and thyroid (7). These data suggest that BTGl down-regulation may be a major contributor to tumour development and progression, and that BTG1 expression scores may provide valuable information for assessments to predict disease severity.

In conclusion, we observed that BTGl expression was down-regulated in CRC cell lines and tissues, and was restored after demethylation treatment in CRC cells, suggesting that down-regulation of BTG1 by promoter methylation may be one of the mechanisms contributing to colorectal carcinogenesis. These results corroborate the role of BTG1 as a tumour suppressor. Restoration of BTG1 expression may offer a new therapeutic approach for treating patients with CRC.

\section{Acknowledgements}

This research was supported by the faculty grant of Myongji Hospital (1701-05-03).

\section{References}

1 Jung KW, Won YJ, Oh CM, Kong HJ, Lee DH, Lee KH and Community of Population-Based Regional Cancer Registries: Cancer statistics in Korea: Incidence, mortality, survival, and prevalence in 2014. Cancer Res Treat 49: 292-305, 2017.

2 Oh CM, Won YJ, Jung KW, Kong HJ, Cho H, Lee JK, Lee DH, Lee KH and Community of Population-Based Regional Cancer Registries: Cancer statistics in Korea: incidence, mortality, survival, and prevalence in 2013. Cancer Res Treat 48: 436-450, 2016.
3 Misiakos EP, Karidis NP and Kouraklis G: Current treatment for colorectal liver metastases. World J Gastroenterol 17: 40674075, 2011.

4 Siegel RL, Miller KD, Fedewa SA, Ahnen DJ, Meester RGS, Barzi A and Jemal A: Colorectal cancer statistics, 2017. CA Cancer J Clin 67: 177-193, 2017.

5 Siegel RL, Miller KD and Jemal A: Cancer statistics, 2017. CA Cancer J Clin 67: 7-30, 2017.

6 Agathocleous M and Harris WA: Metabolism in physiological cell proliferation and differentiation. Trends Cell Biol 23: 484492, 2013.

7 Lu YF, Sun GG, Liu Q, Yang CR and Cheng YJ: BTG1 expression in thyroid carcinoma: diagnostic indicator and prognostic marker. Int J Oncol 45: 1574-1582, 2014.

8 Shibata D and Aaltonen LA: Genetic predisposition and somatic diversification in tumor development and progression. Adv Cancer Res 80: 83-114, 2001.

9 Lee EY and Muller WJ: Oncogenes and tumor -suppressor genes. Cold Spring Harb Perspect Biol 2: a003236, 2010.

10 Berthet C, Guehenneux F, Revol V, Samarut C, Lukaszewicz A, Dehay C, Dumontet C, Magaud JP and Rouault JP: Interaction of PRMT1 with BTG/TOB proteins in cell signalling: molecular analysis and functional aspects. Genes Cells 7: 29-39, 2002.

11 Doidge R, Mittal S, Aslam A and Winkler GS: The antiproliferative activity of BTG/TOB proteins is mediated via the CAF1A (CNOT7) and CAF1B (CNOT8) deadenylase subunits of the CCR4-NOT complex. PLoS One 7: e51331, 2012.

12 Rouault JP, Rimokh R, Tessa C, Paranhos G, Ffrench M, Duret L, Garoccio M, Germain D, Samarut J and Magaud JP: BTG1, a member of a new family of antiproliferative genes. EMBO J 11: 1663-1670, 1992.

13 Guehenneux F, Duret L, Callanan MB, Bouhas R, Hayette S, Berthet C, Samarut C, Rimokh R, Birot AM, Wang Q, Magaud JP and Rouault JP: Cloning of the mouse Btg3 gene and definition of a new gene family (the BTG family) involved in the negative control of the cell cycle. Leukemia 11: 370-375, 1997.

14 Corjay MH, Kearney MA, Munzer DA, Diamond SM and Stoltenborg JK: Antiproliferative gene Btgl is highly expressed in apoptotic cells in macrophage-rich areas of advanced lesions in Watanabe heritable hyperlipidemic rabbit and human. Lab Invest 78: 847-858, 1998.

15 Lee H, Cha S, Lee MS, Cho GJ, Choi WS and Suk K: Role of antiproliferative B -cell translocation gene-1 as an apoptotic sensitizer in activation-induced cell death of brain microglia. $\mathrm{J}$ Immunol 171: 5802-5811, 2003.

16 Farioli-Vecchioli S, Mattera A, Micheli L, Ceccarelli M, Leonardi L, Saraulli D, Costanzi M, Cestari V, Rouault JP and Tirone F: Running rescues defective adult neurogenesis by shortening the length of the cell cycle of neural stem and progenitor cells. Stem Cells 32: 1968-1982, 2014.

17 Lin IY, Chiu FL, Yeang CH, Chen HF, Chuang CY, Yang SY, Hou PS, Sintupisut N, Ho HN, Kuo HC and Lin KI: Suppression of the SOX2 neural effector gene by PRDM1 promotes human germ cell fate in embryonic stem cells. Stem Cell Reports 2: 189-204, 2014.

18 Sun GG, Wang YD, Cheng YJ and Hu WN: BTG1 underexpression is an independent prognostic marker in esophageal squamous cell carcinoma. Tumour Biol 35: 97079716, 2014. 
19 Sun GG, Wang YD, Cheng YJ and Hu WN: The expression of BTG1 is down-regulated in nasopharyngeal carcinoma and possibly associated with tumour metastasis. Mol Biol Rep 41: 5979-5988, 2014.

20 Sun GG, Lu YF, Cheng YJ, Yang CR, Liu Q, Jing SW and Han $\mathrm{XC}$ : Expression of BTG1 in hepatocellular carcinoma and its correlation with cell cycles, cell apoptosis, and cell metastasis. Tumour Biol 35: 11771-11779, 2014.

21 Sun GG, Lu YF, Cheng YJ and Hu WN: The expression of BTG1 is down-regulated in NSCLC and possibly associated with tumor metastasis. Tumour Biol 35: 2949-2957, 2014.

22 Sun G, Liu Q, Cheng Y and Hu W: B cell translocation gene 1 reduces the biological outcome of kidney cancer through induction of cell proliferation, cell cycle arrest, cell apoptosis and cell metastasis. Int J Mol Med 35: 777-783, 2015.

23 Liu C, Tao T, Xu B, Lu K, Zhang L, Jiang L, Chen S, Liu D, Zhang X, Cao N and Chen M: BTG1 potentiates apoptosis and suppresses proliferation in renal cell carcinoma by interacting with PRMT1. Oncol Lett 10: 619-624, 2015.

24 Zhu R, Zou ST, Wan JM, Li W, Li XL and Zhu W: BTG1 inhibits breast cancer cell growth through induction of cell cycle arrest and apoptosis. Oncol Rep 30: 2137-2144, 2013.

$25 \mathrm{Kim}$ HS, Yoon G, Do SI, Kim SJ and Kim YW: Downregulation of osteoprotegerin expression as a novel biomarker for colorectal carcinoma. Oncotarget 7: 15187-15199, 2016.

26 Livak KJ and Schmittgen TD: Analysis of relative gene expression data using real-time quantitative PCR and the 2(-Delta Delta C(T)) Method. Methods 25: 402-408, 2001.

27 Zheng HC, Li J, Shen DF, Yang XF, Zhao S, Wu YZ, Takano Y, Sun HZ, Su RJ, Luo JS and Gou WF: BTG1 expression correlates with pathogenesis, aggressive behaviors and prognosis of gastric cancer: a potential target for gene therapy. Oncotarget 6: 19685-19705, 2015.

28 Moon A, Do SI, Kim HS and Kim YW: Down-regulation of osteoprotegerin expression in metastatic colorectal carcinoma predicts recurrent metastasis and poor prognosis. Oncotarget 7: 79319-79326, 2016.

29 Kim HS, Do SI, Noh BJ, Jeong YI, Park SJ and Kim YW: Expression of phosphorylated extracellular signal-regulated kinase at the invasive front of hepatic colorectal metastasis. Oncol Lett 9: 1261-1265, 2015.

30 Kim HS, Park SJ, Lee KY, Park YK and Kim YW: Reduced Raf1 kinase inhibitor protein expression predicts less favorable outcomes in patients with hepatic colorectal metastasis. Oncol Rep 28: 161-171, 2012.

31 Bae GE, Yoon G, Song YJ and Kim HS: High-grade squamous intraepithelial lesion arising adjacent to vulvar lymphangioma circumscriptum: a tertiary institutional experience. Oncotarget 7: 48120-48129, 2016.

32 Do SI, Yoon G, Kim HS, Kim K, Lee H, Do IG, Kim DH, Chae SW and Sohn JH: Increased brahma-related gene 1 expression predicts distant metastasis and shorter survival in patients with invasive ductal carcinoma of the breast. Anticancer Res 36: 4873-4882, 2016.

33 Park CK, Yoon G, Cho YA and Kim HS: Clinicopathological and immunohistochemical characterization of papillary proliferation of the endometrium: A single institutional experience. Oncotarget 7: 39197-39206, 2016.
34 Yoon N, Yoon G, Park CK and Kim HS: Stromal p16 expression is significantly increased in malignant ovarian neoplasms. Oncotarget 7: 64665-64673, 2016.

35 Yoon G, Koh CW, Yoon N, Kim JY and Kim HS: Stromal p16 expression is significantly increased in endometrial carcinoma. Oncotarget 8: 4826-4836, 2017.

36 Sung JY, Na K and Kim HS: Down-regulation of inositol polyphosphate 4-phosphatase type II expression in colorectal carcinoma. Anticancer Res 37: 5525-5531, 2017.

37 Park CK and Kim HS: Clinicopathological characteristics of ovarian sclerosing stromal tumor with an emphasis on TFE3 overexpression. Anticancer Res 37: 5441-5447, 2017.

38 Do SI, Kim HS, Kim K, Lee H, Do IG, Kim DH, Chae SW and Sohn JH: Predictive value of sphingosine kinase 1 expression in papillary thyroid carcinoma. Anticancer Res 37: 5399-5405, 2017.

39 Kim HS, Yoon G, Ryu JY, Cho YJ, Choi JJ, Lee YY, Kim TJ, Choi $\mathrm{CH}$, Song SY, Kim BG, Bae DS and Lee JW: Sphingosine kinase 1 is a reliable prognostic factor and a novel therapeutic target for uterine cervical cancer. Oncotarget 6: 26746-26756, 2015.

40 Kim JY, Do SI, Bae GE and Kim HS: B-cell translocation gene 1 is down-regulated by promoter methylation in ovarian carcinoma. J Cancer 8: 2669-2795, 2017.

41 Kanda M, Oya H, Nomoto S, Takami H, Shimizu D, Hashimoto R, Sueoka S, Kobayashi D, Tanaka C, Yamada S, Fujii T, Nakayama G, Sugimoto H, Koike M, Fujiwara M and Kodera Y: Diversity of clinical implication of B-cell translocation gene 1 expression by histopathologic and anatomic subtypes of gastric cancer. Dig Dis Sci 60: 1256-1264, 2015.

$42 \mathrm{Wu}$ X, Ding N, Hu W, He J, Xu S, Pei H, Hua J, Zhou G and Wang J: Down-regulation of BTG1 by $m i R-454-3 p$ enhances cellular radiosensitivity in renal carcinoma cells. Radiat Oncol 9: 179, 2014.

43 Lu K, Liu C, Tao T, Zhang X, Zhang L, Sun C, Wang Y, Chen S, Xu B and Chen M: MicroRNA-19a regulates proliferation and apoptosis of castration-resistant prostate cancer cells by targeting BTG1. FEBS Lett 589: 1485-1490, 2015.

44 Zhao Y, Gou WF, Chen S, Takano Y, Xiu YL and Zheng HC: BTG1 expression correlates with the pathogenesis and progression of ovarian carcinomas. Int J Mol Sci 14: 19670-19680, 2013.

45 Sheng SH, Zhao CM and Sun GG: BTG1 expression correlates with the pathogenesis and progression of breast carcinomas. Tumour Biol 35: 3317-3326, 2014.

46 Kanda M, Sugimoto H, Nomoto S, Oya H, Hibino S, Shimizu D, Takami H, Hashimoto R, Okamura Y, Yamada S, Fujii T, Nakayama G, Koike M, Fujiwara M and Kodera Y: B-Cell translocation gene 1 serves as a novel prognostic indicator of hepatocellular carcinoma. Int J Oncol 46: 641-648, 2015. 